\title{
Endovascular aneurysm closure during out of office hours is not related to complications or outcome
}

\author{
Jasper H. van Lieshout ${ }^{1}$ (D) - Dagmar Verbaan ${ }^{2} \cdot$ Igor Fischer $^{3} \cdot$ Hendrik-Jan Mijderwijk $^{1} \cdot$ René van den Berg ${ }^{4}$. \\ W. Peter Vandertop ${ }^{2}$. Catharina J.M. Klijn ${ }^{5}$ - Hans J. Steiger ${ }^{1}$ - Joost de Vries ${ }^{6}$ - Ronald H.M.A. Bartels ${ }^{6}$. \\ Kerim Beseoglu ${ }^{1} \cdot$ Hieronymus D. Boogaarts ${ }^{6}$
}

Received: 2 October 2019 / Accepted: 27 December 2019 / Published online: 7 February 2020

(C) The Author(s) 2020

\begin{abstract}
Purpose A possible disadvantage of endovascular occlusion outside work hours is that complex procedures might expose patients to additional risk when performed in a suboptimal setting. In this prospective cohort study, we evaluated whether treatment during out of office hours is a risk factor for per-procedural complications and clinical outcome.

Methods We included 471 endovascular-treated, consecutive aneurysmal subarachnoid hemorrhage patients $(56.6 \pm 13.1,69 \%$ female), from two prospective observational databases which were retrospectively analyzed. Primary outcome was the occurrence of per-procedural complications. Secondary outcomes were good clinical outcome (modified ranking scale $\leq 2$ ) and death at 6-month follow-up. We determined odds ratios (OR) with 95\% confidence intervals (CI) by ordered polytomous logistic regression analysis and adjusted odds ratios (aOR) for age, World Federation of Neurosurgical Societies grade, and time to treatment.

Results Most patients were treated during office hours $(363 / 471 ; 77.1 \%)$. Treatment during out of office hours did not result in an increased risk of per-procedural complications (OR 0.85 (95\% CI $0.53-1.37 ; p=0.51)$. Patients treated during out of office hours displayed similar odds of good clinical outcome and death after 6 months (OR 1.14, 95\% CI 0.68-1.97 and 1.16 95\% CI 0.562.29 , respectively) compared to patients treated during office hours.

Conclusion In our study, endovascular coil embolization during out of office hours did not expose patients to an increased risk of procedural complications or affect functional outcome after 6 months.
\end{abstract}

Keywords Aneurysmal subarachnoid hemorrhage $\cdot$ Cohortstudy $\cdot$ Risk factors in epidemiology $\cdot$ Outcome research $\cdot$ Patient safety

Jasper H. van Lieshout

Jasper.vanLieshout@med.uni-duesseldorf.de

1 Department of Neurosurgery, Medical Faculty, Heinrich-Heine-University, Moorenstraße 5, 40225 Düsseldorf, Germany

2 Neurosurgical Center Amsterdam, Amsterdam University Medical Centers, Amsterdam, the Netherlands

3 Divisions of Informatics and Statistics, Department of Neurosurgery, University Clinic Düsseldorf, Düsseldorf, Germany

4 Departments of Radiology and Nuclear Medicine, Amsterdam University Medical Centers, Amsterdam, the Netherlands

5 Department of Neurology and Donders Institute for Brain, Cognition and Behaviour, Radboud University Medical Center, Nijmegen, the Netherlands

6 Department of Neurosurgery, Radboud University Medical Center, Nijmegen, the Netherlands

\section{Introduction}

Early $(\leq 72 \mathrm{~h})$ aneurysm closure after intracranial aneurysm rupture is critical to prevent aneurysm rebleeding, and its importance is reflected in current guidelines [1,2]. Some studies have found evidence for a benefit of ultra-early $(\leq$ $24 \mathrm{~h}$ ) or even emergency ( $\leq 6 \mathrm{~h}$ ) treatment of ruptured aneurysms to reduce aneurysmal rebleeding rates and to improve clinical outcome [3,4]. The potential benefit of emergency or ultra-early aneurysm treatment has been suggested to be more pronounced for endovascular coiling than for surgical clipping [4].

However, inconsistent results between studies lead to a lack of evidence on whether earlier endovascular treatment actually improves outcome in patients with aneurysmal subarachnoid hemorrhage (aSAH) [5]. As a result, timing of aneurysm closure remains controversial. In some centers, procedures are 
not performed during out of office hours, because of less optimal logistics and expertise, whereas others favor immediate closure of the aneurysm irrespective of the time of presentation. Van Lieshout et al. recently showed a higher perprocedural re-rupture and a higher probability of poor outcome after emergency coiling $(\leq 6 \mathrm{~h})$ in comparison with

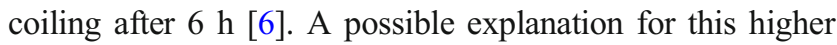
risk may be that patients treated on an emergency basis are more likely to be treated during out of office hours. We hypothesized that patients treated during out of office hours are more likely to experience per-procedural complications and are at higher odds of poor outcome compared to those treated during office hours.

\section{Methods}

\section{Study population and inclusion}

We included 471 consecutive patients with aSAH from two prospective observational databases, at the neurovascular centers from the Radboud University Medical Center (Radboudumc, $n=233$ ), Nijmegen, and the Amsterdam University Medical Centers (location AMC, $n=238$ ), Amsterdam, the Netherlands, between January 2012 and January 2016. Patients from the ultra-early tranexamic acid after subarachnoid hemorrhage (ULTRA) study were not included [7]. Only patients with a ruptured intracranial aneurysm who were treated by endovascular coil embolization were included. The institutional review boards confirmed that the Medical Research Involving Human Subjects Act (WMO) does not apply and that official approval for this study is not required (reference number: 3449 Radboudumc and 17286 for the $\mathrm{AMC})$.

\section{Data collection and organization of the register}

We obtained all data from the Quality Registry Neurosurgery (QRNS) of the Dutch national neurosurgical society (NVvN), a physician-driven prospective national outcome register for aSAH. We obtained the following data: patient characteristics (age, sex), clinical and radiological (Fisher grade, aneurysm size) characteristics, time of ictus and treatment, treatment modality, per-procedural complications, and clinical outcome. Reporting of this study was according to the Strengthening the Reporting of Observational Studies in Epidemiology (STROBE) guidelines for observational studies (supplementary material) [8].

\section{Treatment protocol}

All admitted aSAH patients undergo a standardized treatment protocol, as previously proposed elsewhere $[1,2]$. Both centers aim for aneurysm closure within 8 and at the latest $24 \mathrm{~h}$ after ictus, regardless of aneurysm complexity. For patients in poor clinical condition, World Federation of Neurosurgical Societies (WFNS) grade 5 and timing of treatment were variable and could have been postponed in individual cases.

At the Radboudumc, hybrid vascular neurosurgeons evaluate radiological imaging and make treatment decisions, whereas at the AMC interventional neuroradiologists and vascular neurosurgeons do so in consensus. Both centers provide a 24/7 consultant cover of services. The neuro-interventional specialists who performed the endovascular procedures all had at least 5 years of experience in endovascular treatment of aneurysms and aSAH.

\section{Definitions and outcome parameters}

We defined the time to treatment as the time interval between ictus of hemorrhage and start of endovascular aneurysm closure. The primary outcome of this study was the occurrence of per-procedural complications: aneurysm perforation during the procedure, ischemia attributed to endovascular treatment, thrombus formation, and arterial dissection. The occurrence of any per-procedural complications was scored as a binary event, yes (1) or no (0). We defined office hours from 8:00 a.m. to 5:59 p.m. and out of office hours from 6:00 p.m. to 7:59 a.m.

The secondary outcomes of the study were death and clinical outcome at 6 months after treatment, measured by the modified Rankin scale (mRS) score and determined by a specialized nurse who had not been involved in the patients' treatment. Good clinical outcome was defined as $\mathrm{mRS} \leq 2$ [9]. The mRS was measured with a standardized, validated structured interview.

\section{Data analysis}

Categorical data are presented as numbers (percentages) and continuous variables as mean \pm standard deviation (SD) or median with interquartile range (IQR) depending on the distribution.

We tested categorical data with the Pearson chi-square test and continuous data with the Student's $t$ test for independent observations or the Mann-Whitney test (Wilcoxon signedrank test) as appropriate. We used binary logistic regression analysis to evaluate if time to treatment (during office hours or out of office hours) was associated with per-procedural complications (primary outcome). Ordered polytomous logistic regression analysis (link function: logit) was used to evaluate if time to treatment (during office hours or out of office hours) was associated with clinical outcome after 6 months (secondary outcome). Both analyses were adjusted for age, WNFS 
Table 1 Patient characteristics at baseline distinguished by time of treatment

\begin{tabular}{llll}
\hline & All patients $(n=471)$ & Office hours $(n=363)$ & Out of office hours $(n=107)$ \\
\hline Age, mean \pm SD & $56.6 \pm 13.1$ & $56.9 \pm 12.8$ & $55.7 \pm 14.1$ \\
Female, $n(\%)$ & $326(69.2)$ & $245(67.5)$ & $81(75.7)$ \\
WFNS grade, $n(\%)$ & 462 & 358 & 104 \\
I & $203(43.9)$ & $154(43)$ & $49(47.1)$ \\
II & $100(21.6)$ & $82(22.9)$ & $18(17.3)$ \\
III & $22(4.8)$ & $13(3.6)$ & $9(8.6)$ \\
IV & $58(12.6)$ & $44(12.3)$ & $14(13.5)$ \\
V & $79(17.1)$ & $65(18.2)$ & $14(13.5)$ \\
Fisher grade, $n(\%)$ & 469 & 362 & 107 \\
I & $14(3)$ & $11(3)$ & $3(2.8)$ \\
II & $44(9.4)$ & $31(8.6)$ & $13(12.1)$ \\
III & $103(22)$ & $79(21.8)$ & $24(22.4)$ \\
IV & $308(65.6)$ & $241(66.6)$ & $67(62.6)$ \\
Time to treatment, median $[$ IQR] & $19[10-42]$ & $20[11.5-46.3]$ & $12[7-24]$ \\
\hline
\end{tabular}

Student T-test; *Pearson chi-square test; ¥Mann-Whitney U-test

grade, and time between ictus and treatment, based on subject matter knowledge. $p$ values (two-tailed) and confidence intervals were estimated for all parameters. Patients with missing data or those lost to follow-up were excluded from further analysis for the corresponding analysis.

The type I error was set at 0.05 and the tests were twotailed. For statistical analysis, we used SPSS software version 25.0 (SPSS Institute, Chicago, IL, USA) and the R statistical computing package, $\mathrm{R}$ version 3.4.2 (R Foundation for statistical computing, Vienna, Austria; URL: https://www.Rproject.org/).

\section{Results}

\section{Demographics}

We included 471 patients (mean age 56.6; $\mathrm{SD} \pm 13.1 ; 69 \%$ female). Characteristics of the study population are listed in Table 1. Univariate analysis revealed no differences between the two groups except for time to treatment. Patients treated during out of office hours were treated earlier after ictus compared to those treated during office hours (Table 1). The dataset was incomplete for 19/471 patients (4\%).
Figure 1 This illustrates the distribution of the aneurysms according to the starting time of the endovascular treatment during the day

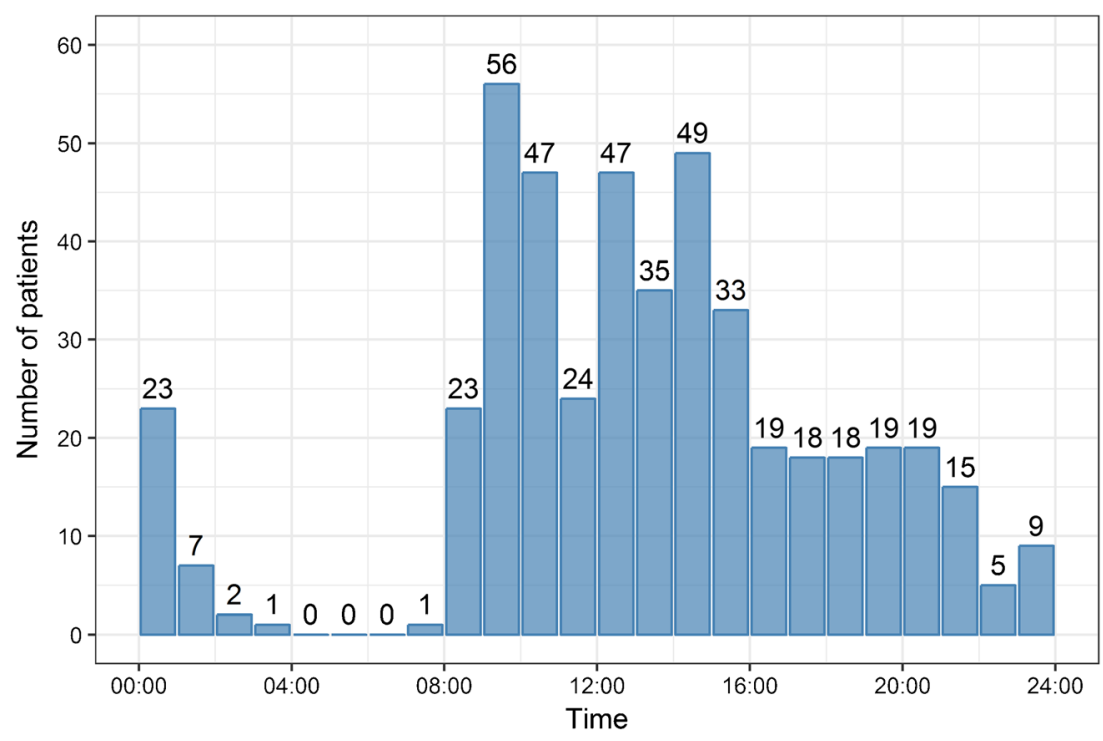


Table 2 Complications of endovascular treatment distinguished by time of treatment

\begin{tabular}{llllll}
\hline & All patients & Office hours & Out of office hours & OR (95\% CI) & aOR (95\% CI) \\
\hline Overall $(n, \%)$ & $55 / 469(11.8)$ & $41 / 364(11.2)$ & $14 / 106(12.2)$ & $0.88(0.53-1.43)$ & $0.85(0.53-1.43)$ \\
Perforation $(n, \%)$ & $12 / 469(2.6)$ & $9 / 364(2.5)$ & $3 / 105(2.9)$ & $0.99(0.26-3.71)$ & $0.94(0.25-3.58)$ \\
Ischemia $(n, \%)$ & $29 / 468(6.2)$ & $25 / 363(6.9)$ & $4 / 105(3.8)$ & $0.60(0.22-1.60)$ & $0.53(0.18-1.6)$ \\
Thrombus $(n, \%)$ & $48 / 468(10.3)$ & $36 / 363(9.9)$ & $12 / 105(11.4)$ & $1.10(0.56-2.17)$ & $1.16(0.58-2.34)$ \\
Dissection $(n, \%)$ & $1 / 459(0.2)$ & $1 / 355(0.3)$ & $0 / 104(0)$ & $0(\mathrm{na})$ & $0(\mathrm{na})$ \\
\hline
\end{tabular}

Logistic regression analysis; na $=$ not applicable

\section{Time of treatment}

We treated more patients during office hours $(363 / 471$; $77.1 \%)$ than during out of office hours $(107 / 471 ; 22.7 \%$, Table 1). In most patients, aneurysm closure was performed between 8:00 and 16:00 as expected. None of the endovascular treatments was started between 4:00 and 7:00, and a minority was started between midnight and 2:00 (Fig. 1). Around a third of the patients with an ictus during office hours were eventually treated during out of office hours $(74 / 253 ; 29.2 \%)$ compared to $15.3 \%$ of the patients with an ictus during out of office hours (33/215).

\section{Per-procedural complications and clinical outcome during out of office hours}

Treatment during out of office hours did not result in an increased risk of per-procedural complications (Table 2). Adjustment for age, WFNS grade, and time to treatment did not change the results (Table 2). Information on clinical outcome 6 months after treatment was available for $96(90.5 \%)$ patients treated during out of office hours and for 327 (89.8\%) patients in the office hour group. Patients treated during out of office hours were at similar odds of good clinical outcome and death at 6 months compared to patients treated during office hours (Table 3 ). There seems to be a bias for the availability of a mRS score after 6 months based on the WFNS grade (Table 4). However, the ORs remained similar after adjustment for age, WFNS grade, and time to treatment (Table 3). The proportional odds assumption was met.

\section{Discussion}

In this study, endovascular coil embolization during out of office hours was not associated with an increased risk of treatment-related complications, poor functional outcome, or death after 6 months.

Healthcare professionals who treat patients with ischemic stroke are often the same who treat patients with aSAH. As a result, resources for the diagnosis and treatment of aSAH are available round-the-clock in many neurovascular centers. Nevertheless, a survey among German hospitals involved in treatment of aSAH showed that the majority of hospitals $(98 \%)$ does not provide immediate aneurysm closure at night, be it by either endovascular or neurosurgical treatment [10]. Moreover, most hospitals do not start treatment later than 20:00. Both human and situational factors may cause decreased quality of care during nighttime, and indeed task performance on manual monitoring tasks has been found worse at night $[11,12]$. It is therefore surmised that aneurysm repair during out of office hours increases the risk of treatmentrelated complications, based upon translation of results from nightly treatment of other diseases, although so far this has not been studied explicitly for endovascular closure of ruptured cerebral aneurysms $[10,13,14]$.

Our results stand in contrast with findings from the before mentioned reports but recapitulates the results of a previous study, which show that nighttime surgery is not independently associated with higher intraoperative risk of complications [15].

Strengths of our study include the prospective data collection representing a homogenous national healthcare setting and a high follow-up rate. However, there are

Table 3 Functional outcome after 6 months

\begin{tabular}{llllll}
\hline & All patients $(n=424)$ & Office hours $(n=327)$ & Out of office hours $(n=97)$ & OR (95\% CI) & aOR (95\% CI) \\
\hline $\mathrm{mRS}, n(\%)$ & & & & \\
$\mathrm{mRS} \leq 2$ & $290(68.4)$ & $222(67.9)$ & $68(70.1)$ & $1.11(0.65-1.90)$ & $1.14(0.68-1.97)$ \\
$\mathrm{mRS}=6$ & $56(13.2)$ & $42(12.8)$ & $14(14.6)$ & $1.09(0.63-1.85)$ & $1.16(0.56-2.29)$ \\
\hline
\end{tabular}

Logistic regression analysis 
Table 4 Patient characteristics at baseline distinguished availability of mRS score after 6 months

\begin{tabular}{llll}
\hline & All patients $(n=471)$ & mRS available $(n=424)$ & $\begin{array}{l}\text { no mRS available } \\
(n=47)\end{array}$ \\
\hline Age, mean \pm SD & $56.6 \pm 13.1$ & $56.5 \pm 12.9$ & $57.3 \pm 14.8$ \\
Female, $n(\%)$ & $327(69.2)$ & $298(70.2)$ & $29(61.7)$ \\
WFNS grade, $n(\%)$ & 463 & 419 & 44 \\
I & $204(43.9)$ & $179(42.2)$ & $25(56.8)$ \\
II & $100(21.6)$ & $95(22.4)$ & $5(11.4)$ \\
III & $22(4.8)$ & $22(5.2)$ & $0.415)$ \\
IV & $58(12.6)$ & $49(11.6)$ & $9(20.5)$ \\
V & $79(17.1)$ & $74(17.5)$ & $5(11.4)$ \\
Fisher grade, $n(\%)$ & 469 & 422 & 47 \\
I & $14(3)$ & $14(3.3)$ & $0.046^{*}$ \\
II & $44(9.4)$ & $41(9.7)$ & $3(6.4)$ \\
III & $103(22)$ & $90(21.2)$ & $13(27.7)$ \\
IV & $308(65.6)$ & $241(56.8)$ & $31(66)$ \\
Time to treatment, median $[$ IQR] & $19[10-42]$ & $18.5[9.5-41]$ & $24[12.5-52]$ \\
\hline
\end{tabular}

Student T-test; *Pearson chi-square test; ¥Mann-Whitney U-test

several limitations that need to be addressed. Since very few patients were treated between midnight and 8:00, it is challenging to draw conclusions on the risks of treatment during those early hours. Since we started treatment between 1:00 and 8:00 a.m. in just 11 patients, our results seem valid for a treatment onset time up to 1 a.m. Our data suggest that not the clinical status but the hour of day determines delay of endovascular aneurysm closure. As a result, none of the endovascular treatments started between 4:00 and 7:00, and only few treatments started between midnight and 4:00. Despite the collaboration between two referral centers, the number of patients with procedural complications was small, and not all potential relevant factors were collected in the prospective database, such as blood pressure on admission and during the procedure. Due to the disbalance of the number of patients between the groups, our study may be underpowered and limited by selection bias. Moreover, possible imprecision in determining time of ictus could have influenced the registered time to treatment and might therefore have altered our findings. Finally, this study was observational, and not a randomized comparison of patients presenting during out of office hours treated immediately or delayed, so firm conclusions cannot be drawn on the optimal timing of treatment.

Emergency aneurysm closure provides a theoretical benefit by eliminating the early risk of rebleeding. However it is questioned if in emergency aneurysm occlusion, the theoretical benefit of early repair might be offset by an increased risk of per-procedural complication of rerupture [6]. The overall benefit of early endovascular aneurysm closure on outcomes is unclear, but aneurysm obliteration during out of office hours does not seem to have a negative impact on outcome and is therefore not an important argument against immediate treatment [5]. Due to the marked increased burden on resources, routine aneurysm closure during out of office hours would need a clear justification. Future efforts should focus on establishing whether emergency aneurysm closure improves patient outcome. If the beneficial effect of emergency aneurysm occlusion by reducing spontaneous rebleeding rates outweighs the higher rate of procedural aneurysm re-rupture, aneurysm closure after aSAH should be regarded as a neurological emergency similar to ischemic stroke [6].

\section{Conclusion}

In this study, endovascular coil embolization during out of office hours was not associated with an increased risk of treatment-related complications, poor functional outcome, or death after 6 months. Few patients were treated at night, but we could not confirm the perceived notion that endovascular aneurysm treatment during out of office hours is associated with a higher risk of procedural complications.

Acknowledgments Open Access funding provided by Projekt DEAL. The authors thank Harriette Peterson (Radboud University Medical Center Nijmegen, Department of Neurosurgery) for the meticulous prospective data entry.

Funding information No targeted funding. 


\section{Compliance with ethical standards}

Conflict of interest Jasper Hans van Lieshout - Reports no disclosures Dagmar Verbaan - Reports no disclosures

Hendrik-Jan Mijderwijk - Reports no disclosures

Igor Fischer - Reports no disclosures

René van den Berg - Consultant for Cerenovus

Peter Vandertop - Reports no disclosures

Catharina Klijn - CJMK was supported by a clinical established investigator grant of the Dutch Heart Foundation (grant number 2012 T077), and an Aspasia grant from The Netherlands Organisation for Health Research and Development (ZonMw grant 015.008.048).

Hans-Jakob Steiger - Reports no disclosures

Joost de Vries: Consultant for Stryker Neurovascular

Ronald Bartels - Reports no disclosures

Kerim Beseoglu - Reports no disclosures

Hieronymus Boogaarts: Reports no disclosures

Ethical approval All procedures performed in the studies involving human participants were in accordance with the ethical standards of the institutional and/or national research committee and with the 1964 Helsinki Declaration and its later amendments or comparable ethical standards.

Informed consent Ethical approval for this study was waived by the institutional review boards because the Medical Research Involving Human Subjects Act (WMO) does not apply and therefore official approval for this study is not required.

Open Access This article is licensed under a Creative Commons Attribution 4.0 International License, which permits use, sharing, adaptation, distribution and reproduction in any medium or format, as long as you give appropriate credit to the original author(s) and the source, provide a link to the Creative Commons licence, and indicate if changes were made. The images or other third party material in this article are included in the article's Creative Commons licence, unless indicated otherwise in a credit line to the material. If material is not included in the article's Creative Commons licence and your intended use is not permitted by statutory regulation or exceeds the permitted use, you will need to obtain permission directly from the copyright holder. To view a copy of this licence, visit http://creativecommons.org/licenses/by/4.0/.

\section{References}

1. Steiner T, Juvela S, Unterberg A, Jung C, Forsting M, Rinkel G, European Stroke O (2013) European Stroke organization guidelines for the management of intracranial aneurysms and subarachnoid haemorrhage. Cerebrovasc Dis 35(2):93-112. https://doi.org/10. $1159 / 000346087$

2. Connolly ES Jr, Rabinstein AA, Carhuapoma JR, Derdeyn CP, Dion J, Higashida RT, Hoh BL, Kirkness CJ, Naidech AM, Ogilvy CS, Patel AB, Thompson BG, Vespa P, American Heart Association Stroke C, Council on Cardiovascular R, Intervention, Council on Cardiovascular N, Council on Cardiovascular S, Anesthesia, Council on Clinical C (2012) Guidelines for the management of aneurysmal subarachnoid hemorrhage: a guideline for healthcare professionals from the American Heart Association/ American Stroke Association. Stroke 43(6):1711-1737. https:// doi.org/10.1161/STR.0b013e3182587839

3. Park J, Woo H, Kang DH, Kim YS, Kim MY, Shin IH, Kwak SG (2015) Formal protocol for emergency treatment of ruptured intracranial aneurysms to reduce in-hospital rebleeding and improve clinical outcomes. J Neurosurg 122(2):383-391. https://doi. org/10.3171/2014.9.JNS131784

4. Phillips TJ, Dowling RJ, Yan B, Laidlaw JD, Mitchell PJ (2011) Does treatment of ruptured intracranial aneurysms within 24 hours improve clinical outcome? Stroke 42(7):1936-1945. https://doi. org/10.1161/STROKEAHA.110.602888

5. Rawal S, Alcaide-Leon P, Macdonald RL, Rinkel GJ, Victor JC, Krings T, Kapral MK, Laupacis A (2017) Meta-analysis of timing of endovascular aneurysm treatment in subarachnoid haemorrhage: inconsistent results of early treatment within 1 day. J Neurol Neurosurg Psychiatry 88(3):241-248. https://doi.org/10.1136/ jnnp-2016-314596

6. van Lieshout JH, Verbaan D, Donders R, van den Berg R, Vandertop PWP, Klijn CJM, Steiger HJ, de Vries J, Bartels R, Beseoglu K, Boogaarts HD (2019) Periprocedural aneurysm rerupture in relation to timing of endovascular treatment and outcome. J Neurol Neurosurg Psychiatry 90(3):363-365. https://doi. org/10.1136/jnnp-2018-318090

7. Germans MR, Post R, Coert BA, Rinkel GJ, Vandertop WP, Verbaan D (2013) Ultra-early tranexamic acid after subarachnoid hemorrhage (ULTRA): study protocol for a randomized controlled trial. Trials 14:143. https://doi.org/10.1186/1745-6215-14-143

8. von Elm E, Altman DG, Egger M, Pocock SJ, Gotzsche PC, Vandenbroucke JP, Initiative S (2007) Strengthening the reporting of observational studies in epidemiology (STROBE) statement: guidelines for reporting observational studies. BMJ 335(7624): 806-808. https://doi.org/10.1136/bmj.39335.541782.AD

9. Weisscher N, Vermeulen M, Roos YB, de Haan RJ (2008) What should be defined as good outcome in stroke trials; a modified Rankin score of 0-1 or 0-2? J Neurol 255(6):867-874. https://doi. org/10.1007/s00415-008-0796-8

10. Janssen H, Berlis A, Lutz J, Thon N, Bruckmann H (2017) State of practice: endovascular treatment of acute aneurysmal SAH in Germany. AJNR Am J Neuroradiol 38(8):1574-1579. https://doi. org/10.3174/ajnr.A5260

11. Cao CG, Weinger MB, Slagle J, Zhou C, Ou J, Gillin S, Sheh B, Mazzei W (2008) Differences in day and night shift clinical performance in anesthesiology. Hum Factors 50(2):276-290. https://doi. org/10.1518/001872008X288303

12. Cortegiani A, Gregoretti C, Neto AS, SNT H, Ball L, Canet J, Hiesmayr M, Hollmann MW, Mills GH, MFV M, Putensen C, Schmid W, Severgnini P, Wrigge H, Gama de Abreu M, Schultz MJ, Pelosi P, Las Vegas Investigators tPN, the Clinical Trial Network of the European Society of A (2019) Association between night-time surgery and occurrence of intraoperative adverse events and postoperative pulmonary complications. Br J Anaesth 122(3): 361-369. https://doi.org/10.1016/j.bja.2018.10.063

13. Elshove-Bolk J, Ellensen VS, Baatrup G (2010) Logistics and outcome in urgent and emergency colorectal surgery. Colorectal Dis 12(10 online):e255-e259. https://doi.org/10.1111/j.1463-1318. 2009.02120.x

14. Rothschild JM, Keohane CA, Rogers S, Gardner R, Lipsitz SR, Salzberg CA, Yu T, Yoon CS, Williams DH, Wien MF, Czeisler CA, Bates DW, Landrigan CP (2009) Risks of complications by attending physicians after performing nighttime procedures. JAMA 302(14):1565-1572. https://doi.org/10.1001/jama.2009.1423

15. Eskesen TG, Peponis T, Saillant N, King DR, Fagenholz PJ, Velmahos GC, Kaafarani HMA (2018) Operating at night does not increase the risk of intraoperative adverse events. Am J Surg 216(1):19-24. https://doi.org/10.1016/j.amjsurg.2017.10.026

Publisher's note Springer Nature remains neutral with regard to jurisdictional claims in published maps and institutional affiliations. 